\title{
Effects of tongxinluo on angiogenesis in the carotid adventitia of hyperlipidemic rabbits
}

\author{
JIANHUA ZHOU, BINGFENG CAO, WEIPING JU, SEN LIU, JIANGUANG SONG and LIXIA LIU
}

\author{
Department of Cardiology, Second Ward, Shandong Weihai Central Hospital, Weihai, Shandong 264400, P.R. China
}

Received June 24, 2015; Accepted July 4, 2016

DOI: $10.3892 / \mathrm{mmr} .2016 .5675$

\begin{abstract}
Atherosclerosis, as a common arterial disease with high morbidity rate, is reported to be closely associated with adventitia angiogenesis. The present study aimed to investigate the effect of tongxinluo (TXL) on angiogenesis in the carotid adventitia of hyperlipidemic rabbits and the underlying mechanism. A total of 90 experimental rabbits were randomly assigned into the following six groups $(n=15$ per group): Normal group, model group, low-dose TXL group, moderate-dose TXL group, high-dose TXL group and atorvastatin group. The normal group was fed with a standard diet. The model and treatment groups were on a high cholesterol diet for 4 weeks. The serum lipid level of the model group was significantly higher compared with the normal group. TXL serum lipid level compared with the model group. Hematoxylin and eosin, and CD31 staining demonstrated that TXL inhibited adventitia angiogenesis in a dose-dependent manner. The dihydroethidium probe and fluorescence in situ hybridization results indicated that TXL reduced $\mathrm{O}_{2}^{-}$level and positive signal of gp91phox and p22phox mRNA in adventitia. Reverse transcription-polymerase chain reaction and western blot analysis determined that TXL treatment significantly downregulated the expression levels of the gp91phox, p22phox genes and the vascular endothelial growth factor (VEGF), vascular endothelial growth factor receptor-2 (VEGFR-2) proteins compared with the model group. TXL exhibited a dose-dependent inhibitory effect on angiogenesis in the carotid adventitia of hyperlipidemic rabbits. This may be associated with the downregulation of reactive oxygen species generation in the adventitia and the suppression of VEGF/VEGFR-2 expression.
\end{abstract}

Correspondence to: Mrs. Lixia Liu, Department of Cardiology, Second Ward, Shandong Weihai Central Hospital, 3 Mishan Road, Wendeng, Weihai, Shandong 264400, P.R. China

E-mail: yushoush@126.com

Key words: vasa vasorum, angiogenesis, atherosclerosis, oxidative stress, tongxinluo

\section{Introduction}

With the rapid development of the global economy and the rising quality of life, the morbidity rate of atherosclerosis (AS) is increasing. Major cardiovascular and cerebrovascular diseases, including coronary heart disease (CHD), stroke and sudden death have become the leading cause of mortality worldwide. The progression of AS has been frequently investigated and various hypotheses on its pathogenesis have been proposed. However, the exact etiology and mechanism of this disease has not been fully elucidated. The hypothesis of 'inside-out' inflammation has been proposed by Ross, which suggests that AS is an 'inside-out' vascular inflammatory response (1-3). However, this theory fails to fully clarify the pathogenesis of AS. Furthermore, the anti-inflammatory and lipid-reducing statin-based treatments, suggested therapy based on this theory may achieve an efficacy rate of 30-40\% in the treatment of atherosclerotic cardio-cerebral vascular diseases (4).

Previous studies have demonstrated that tunica adventitia contributes to the formation of the intimal lesion of AS. According to a previous study, angiogenesis in the arterial wall adventitia occurs at the stage of hyperlipidemia, prior to endothelial dysfunction in great vessels (5). Additionally, clinical and experimental studies have demonstrated that neovascularization is closely associated with the development of AS and that the degree of proliferation of the vasa vasorum in adventitia was positively associated with microvascular network expansion in the plaques $(6,7)$. A previous study, which investigated the effect of the local application of pro-angiogenic, fibroblast growth factor 2 in the abdominal aortic adventitia of $\mathrm{ApoE}^{---}$mice, determined that the adventitia angiogenesis occurred prior to the development of AS and the quantity of vasa vasorum was proportional to the size of the plaque (8). In a pig model of hyperlipidemia, the use of thalidomide, an inhibitor of angiogenesis, effectively reduced the expression of the pro-angiogenic vascular endothelial growth factor (VEGF) protein, inhibited adventia angiogenesis in the coronary artery and delayed neointima formation (9). Additionally, previous studies have also suggested that inhibiting angiogenesis in the vascular wall may effectively impede AS (10-12). Thus, inhibiting neovascularization may represent a novel treatment for AS.

Tongxinluo (TXL) is a traditional Chinese medicine (TCM), which is composed of 12 Chinese medicines and 
herbs, including ginseng, ground beetle (usually rich in chitin), leech, scorpion, centipede, cicada slough, red peony root, spina date seed, dalbergia wood and borneol. Its primary active ingredients include paeoniflorin, ginsenoside Rg1, ginsenoside Rb1 and jujubosides A and B (13). Approved by registration at the State Food and Drug Administration of China, in 1996, TXL has been used widely for the clinical treatment of CHD, angina and ischemic stroke. Evidence has previously demonstrated that TXL protects the cardiovascular system and may reduce blood lipid levels, inhibit inflammation and oxidation, effectively attenuate plaque formation, stabilize vulnerable plaque and prevent plaque rupture (14). However, the molecular mechanism behind plaque stabilization and the AS-inhibitory effects of TXL remain unclear. This may be associated with the inhibitory effect on adventitia angiogenesis. The present study established a rabbit model of hyperlipidemia to investigate the effects of TXL on angiogenesis in the early stage of AS and the underlying molecular mechanisms.

\section{Materials and methods}

Experimental animals. The present study was approved by the Animal Ethics Committee of Shandong Wendeng Central Hospital (Weihai, China). A total of 90 common New Zealand male white rabbits, aged 3-4 months, with an average weight of $2.0 \pm 0.2 \mathrm{~kg}$ (specific pathogen-free grade, certificate no. 2009009), were purchased from the Experimental Animal Center of Shandong Luye Pharmaceutical Co., Ltd. (Yantai, China). The animals were housed in individual cages at a constant temperature $\left(20-36^{\circ} \mathrm{C}\right)$ and humidity (40-70\%).

Modeling, grouping and medication. All experimental animals were fed adaptively for 7 days and then randomly assigned to the following treatment groups ( $n=15$ per group): Normal group, model group, low-dose TXL group (TXL-L), moderate-dose TXL group (TXL-M), high-dose TXL group (TXL-H) and atorvastatin group. The normal group was fed with a standard diet for the duration of the study. The model group and medicine-treated groups were fed with a high cholesterol diet (1\% cholesterol, 5\% lard, $7.5 \%$ yolk powder and $86.5 \%$ basal feed). The feed was processed by the Hebei Laboratory Animal Center (Hebei, China). Each rabbit was fed with $120 \mathrm{~g}$ of feed each day for 4 weeks.

The TXL-L, TXL-M and TXL-H groups were given TXL superfine powder at $0.15,0.30$ and $0.6 \mathrm{~g} / \mathrm{kg} / \mathrm{day}$; Yiling Pharmaceutical Co., Inc., Shijiazhuang, China), respectively. Considering the strong angiogenesis regulation effects of atorvastatin, the atorvastatin group was used as the positive control and was administered with atorvastatin by oral gavage at $2.50 \mathrm{mg} / \mathrm{kg} /$ day (Lipitor Pfizer Pharmaceuticals, Ltd., Dalian, China). The treatments, at the aforementioned concentrations, were prepared and administered to the rabbits by oral gavage as $3 \mathrm{ml} / \mathrm{kg}$ body weight. Gavage administration was initiated subsequently to grouping. The normal group and model groups were treated with $0.5 \%$ carboxy-methyl-cellulose sodium solution according to weight standard of $3 \mathrm{ml} / \mathrm{kg}$. The experimental period was 4 weeks.

Specimen collection. Specimen collection for each group was performed at the end of the 4-week experimental period. Prior to sampling, animals were fasted for $12 \mathrm{~h}$. All animals were sacrificed after an injection with $3 \%$ pentobarbital sodium $(1 \mathrm{ml} / \mathrm{kg}$ ) through the ear vein. Fasting blood samples were obtained via the abdominal aorta of the rabbits and used to determine the lipid levels in the blood serum. Subsequent to the removal of the right common carotid artery, which was washed in ice-cold normal saline, the attached connective tissues were removed with caution. One segment of the collected sample was fixed in $10 \%$ neutral formaldehyde solution, then embedded in paraffin for hematoxylin and eosin (HE) staining, immunohistochemical staining and in situ hybridization experiments. The procedure was performed under aseptic conditions. The remaining segment was frozen in liquid nitrogen for subsequent molecular biological assays. Additionally, the right common carotid arteries were removed from 1 in 3 randomly selected rabbits in each group and immediately processed into frozen sections in order to determine $\mathrm{O}_{2}^{-}$levels.

Determination of blood lipid levels. Enzymatic colorimetry and an automatic biochemical analyzer were used to detect serum total cholesterol (TC), triglyceride (TG), low density lipoprotein (LDL) and high density lipoprotein (HDL) levels. Determination of these physiological and biochemical parameters were measured by colorimetric enzyme kits, according to the manufacturer's protocol (Nanjing Jiancheng Bioengineering Institute, Nanjing, China)

Morphological analysis and immunohistochemical staining. Subsequent to fixing of the specimen, $5 \mu$ m-thick paraffin sections were prepared for HE staining and CD31 immunohistochemical staining. The EnVision immunohistochemical staining method was used to determine localization and expression of CD31 in carotid artery tissue. Following dewaxing, the $5 \mu \mathrm{m}$-thick carotid specimen section was placed in $3 \% \mathrm{H}_{2} \mathrm{O}_{2}$ solution to block endogenous peroxidase. The sample tissue was incubated with the CD31 primary antibody (cat. no. ab958; Abcam, Cambridge, UK) diluted to 1:200 with 5\% BSA at $4^{\circ} \mathrm{C}$ overnight. Subsequently, the horseradish peroxidase (HRP)-labeled secondary antibody (cat. no. ab6785; Abcam; dilution, 1:1,000) was added to detect the primary antibody and 3,3'-diaminobenzidine color developing was performed. Hematoxylin was used to stain the cell nuclei. Finally, the tissue section was dehydrated, covered and observed under an optical microscope.

The microvessel density (MVD) count method, as described by Weidner et al (15), was used for MVD quantification analysis of the labeled CD31. Vascular endothelial cells were stained brownish-yellow by the secondary antibody conjugated to CD31. The isolated endothelial cell clusters were scored as a microvessels if they demonstrated a clear boundary with adjacent cells and the surrounding connective tissue, irrespective of the existence of vessel lumen. Initially, the whole section was observed under a microscope at $\mathrm{x} 40$ magnification in order to select for a high-MVD concentration region. Subsequently, the microvessel quantities (Q) were counted in 5 high-power fields at a magnification of $\mathrm{x} 400$ in a microscopic visual field and the cross-sectional area $\left(\mathrm{A} ; \mathrm{mm}^{2}\right)$ of the vascular wall was determined. The MVD $\left(\mathrm{n} / \mathrm{mm}^{2}\right)$ was calculated using the following formula: $\mathrm{MVD}=\mathrm{Q} / \mathrm{A}$. The 
average of the MVDs in the five high-power fields was used as the MVD of the sample.

Detection of superoxide anion. In accordance with a previous study (16), dihydroethidium (DHE) fluorescence probe was used to detect $\mathrm{O}_{2}(\bullet-)$ and evaluate the level of reactive oxygen species (ROS) in the adventitia. Fresh tissues $(20 \mathrm{~mm})$ of common right carotid artery were taken from 1 in 3 animals randomly selected from each group, quickly placed in optimal cutting temperature embedding medium, frozen and cut into $30 \mu \mathrm{m}$-thick sections by a freezing microtome. The DHE probe was dissolved in dimethyl sulfoxide and then diluted with phosphate-buffered saline to a working solution of $10 \mu \mathrm{mol} / \mathrm{l}$. DHE probe working solution $(100 \mu \mathrm{l})$ was added onto the surface of the section, which was then promptly placed in a light-tight wet box for $30 \mathrm{~min}$ at $37^{\circ} \mathrm{C}$, in order to oxidize DHE and generate ethidium bromide (EB). The EB binds to DNA in the cell nucleus to produce red fluorescence under UV light. Subsequently, unreacted probes were washed away and the sample was observed under a fluorescence microscope. Under excitation at a wavelength of $490 \mathrm{~nm}$ and an emission wavelength of $520 \mathrm{~nm}$, the signal intensity of the red fluorescence was observed. Microscopic imaging analysis was adopted for image acquisition. Image-Pro Plus 6.0 (Media Cybernetics, Inc., Rockville, MD, USA) was used for the quantitative analysis of the fluorescence intensity of the image.

Fluorescence in situ hybridization. For the localization of the carotid artery the nicotinamide adenine dinucleotide phosphate $[\mathrm{NAD}(\mathrm{P}) \mathrm{H}]$ oxidase subunits p22phox and gp91phox mRNA were detected using in situ hybridization with fluorescein isothiocyanate (FITC)-conjugated DNA probes. The sequences of the probes used were as follows: p22phox, 5'-FITC+CCAGGAGCTTCAGCACGGCGGTCA GGTAGCG-3'; and gp91phox, 5'-FITC+ACCATTTTATGA AAAGTGAGATTT CTG-3'. Carotid arterial tissue was fixed in $10 \%$ neutral formaldehyde solution an cut into $5 \mu \mathrm{m}$-thick paraffin sections. Subsequent to dewaxing and air drying, the sections were soaked in proteinase $\mathrm{K}$ reaction solution (100 mmol/1 Tris-HCL, $50 \mathrm{mmol} / \mathrm{l}$ EDTA and $1 \mu \mathrm{g} / \mathrm{ml}$ proteinase $\mathrm{K}$ ), incubated in a $37^{\circ} \mathrm{C}$ water bath for $20 \mathrm{~min}$, rinsed 3 times with saline sodium citrate (SSC) buffer and dehydrated through an alcohol gradient. The sections were then placed in a staining jar containing denaturing solution (70\% methanamide, 2X SSC and $0.1 \mathrm{mmol} / 1$ EDTA) and incubated at $75^{\circ} \mathrm{C}$ for $8 \mathrm{~min}$, simultaneously hybridization solution containing RNA probe (300 $\mu 1$ formamide, $12 \mu 1$ 50X Denhardt's solution, $120 \mu 150 \%$ dextran sulfate, $10 \mu \mathrm{g} / \mu \mathrm{l}$ yeast tRNA, $10 \mu \mathrm{l}$ RNA probe and $49 \mu \mathrm{l}$ RNase-free deionized water) was incubated at $75^{\circ} \mathrm{C}$ for $8 \mathrm{~min}$. The specimen area of each slide was covered with $50 \mu$ l hybridization mix solution, placed into a wet box and subjected to hybridization in a $42^{\circ} \mathrm{C}$ oven overnight. Subsequent to hybridization, the slide was eluted, dehydrated through an alcohol gradient and air-dried. Signals were observed under a fluorescence microscope.

Reverse transcription-polymerase chain reaction (RT-PCR) analysis. The total RNA was extracted using RNAiso Plus
TRIzol kit (Takara Bio, Inc., Dalian, China), according to the manufacturer's protocol. The cDNA was synthetized using PrimeScript RT reagent kit with gDNA Eraser (Takara Bio., Inc.) in a final volume of $20 \mu \mathrm{l}$. The reverse transcription was programmed at $42^{\circ} \mathrm{C}$ for $2 \mathrm{~min}$, followed by $37^{\circ} \mathrm{C}$ for $15 \mathrm{~min}$ and $85^{\circ} \mathrm{C}$ for $5 \mathrm{sec}$. Expression of the NAD $(\mathrm{P}) \mathrm{H}$ oxidase subunits p22phox and gp91phox mRNA was determined by RT-PCR. Primers were synthesized by Generay Biotech Co., Ltd. (Shanghai, China). Primers for amplification of p22phox, gp91phox and GAPDH were designed using published rabbit phagocyte sequences to amplify fragments of 77, 442 and $104 \mathrm{bp}$, respectively. Primer sequences were as follows: p22phox (NM_001082099), sense 5'-GTCGTG TGACTGCCACCTCTGA-3', antisense 5'-GATGTCCACTGT GTTTACTGCAGG-3'; and gp91phox (NM_001082100.1), sense 5'-CCAGTGCGTGCTGCTCAACAAG-3', antisense 5'-GTACAATTCGTTCAGCTCCATGGATG-3'. GAPDH (NM_001082253.1), used as an internal control, was amplified by the use of sense 5'-AGAGCACCAGAG GAGGACG-3' and antisense, 5'-TGGGATGGAAACTGT GAAGAG-3' primers. The thermocycling conditions were as follows: $50^{\circ} \mathrm{C}$ for $30 \mathrm{~min}, 94^{\circ} \mathrm{C}$ for $2 \mathrm{~min}, 94^{\circ} \mathrm{C}$ for $30 \mathrm{sec}, 55^{\circ} \mathrm{C}$ for $30 \mathrm{sec}$, and $72^{\circ} \mathrm{C}$ for $2 \mathrm{~min}$, followed by final extension at $72^{\circ} \mathrm{C}$ for $5 \mathrm{~min}$. A total of 40 cycles were used for $\mathrm{p} 22$ phox and gp91phox amplification.

Western blot analysis. Three samples from each group were analyzed. Carotid artery tissue (100 mg) was homogenized with tissue lysate solution. Subsequent to homogenization, centrifugation was performed at $12,000 \mathrm{x}$ g at $4^{\circ} \mathrm{C}$ to separate the supernatant. Protein concentration was measured by the Coomassie brilliant blue method. Equivalent protein extracts $(30 \mu \mathrm{g})$ were obtained from each group, separated by $10 \%$ sodium dodecyl sulfate polyacrylamide gel and transferred onto polyvinylidene difluoride membrane via electrotransfer, blocked with $5 \%$ skim milk powder, reacted with VEGF antibody (cat. no. ab1316; Abcam, Cambridge, UK) and vascular endothelial growth factor receptor-2 (VEGFR-2) antibody (cat. no. b334966; Biorbyt, Cambridge, UK) and were incubated at $4{ }^{\circ} \mathrm{C}$ overnight and washed, and then reacted with corresponding HRP-labeled secondary antibody (cat. nos. ab6785 and ab6881; dilution, 1:5,000 and 1:10,000; both from Abcam) at room temperature for $90 \mathrm{~min}$. Subsequently, the membrane was washed and enhanced chemiluminescence was used for color development. Another identical membrane was prepared with $\beta$-actin antibody diluents (cat. no. ab8227; dilution 1:1,000) as an internal control and processed by the aforementioned method. The Gel Doc 2000 UVP gel imaging system (UVP, LLC, Upland, CA, USA) was used for film scanning. Labwork (version 4.6) Analysis software (Media Cybernetics, Inc., Rockville, MD, USA) was used to perform gray value analysis of the target band and $\beta$-actin internal control. The ratio of 'gray value of targeted protein signal intensity' and 'gray value of internal control $\beta$-actin signal intensity' was calculated and represents the relative expression level of the protein of interest.

Statistical analysis. The statistical analysis software SPSS version 19.0 (IBM SPSS, Armonk, NY, USA) was used for data analysis. Continuous variable data were represented by 
Table I. Changes in the blood lipid levels of the various treatment groups.

\begin{tabular}{lcccc}
\hline Group & TC & TG & LDL & HDL \\
\hline Normal & $4.66 \pm 1.09$ & $1.89 \pm 0.55$ & $0.68 \pm 0.23$ & $1.05 \pm 0.40$ \\
Model & $58.75 \pm 15.22^{\mathrm{a}}$ & $4.23 \pm 1.26^{\mathrm{a}}$ & $17.28 \pm 4.27^{\mathrm{a}}$ & $2.98 \pm 0.75^{\mathrm{a}}$ \\
TXL-L & $58.67 \pm 13.72$ & $3.87 \pm 0.99$ & $15.90 \pm 4.43$ & $3.03 \pm 0.78$ \\
TXL-M & $27.93 \pm 8.02^{\mathrm{c}}$ & $2.83 \pm 0.96^{\mathrm{c}}$ & $9.48 \pm 2.11^{\mathrm{b}}$ & $3.32 \pm 0.61$ \\
TXL-H & $25.71 \pm 10.24^{\mathrm{c}}$ & $2.84 \pm 0.52^{\mathrm{c}}$ & $7.80 \pm 3.45^{\mathrm{c}}$ & $4.21 \pm 0.80^{\mathrm{c}}$ \\
Atorvastatin & $17.43 \pm 5.49^{\mathrm{c}}$ & $2.69 \pm 0.75^{\mathrm{c}}$ & $7.26 \pm 1.41^{\mathrm{c}}$ & $4.31 \pm 0.99^{\mathrm{c}}$ \\
\hline
\end{tabular}

${ }^{\mathrm{a}} \mathrm{P}<0.01$ vs. normal group. ${ }^{\mathrm{b}} \mathrm{P}<0.05,{ }^{\mathrm{c}} \mathrm{P}<0.01$ vs. model group. $\mathrm{n}=8$. Values are mmol $/ 1, \mathrm{x} \pm \mathrm{s}$. TC, total cholesterol; TG, triglyceride; LDL-C, low-density lipoprotein; HDL-C, high-density lipoprotein; TXL, tongxinluo; L, low; M, medium; H, high.

A
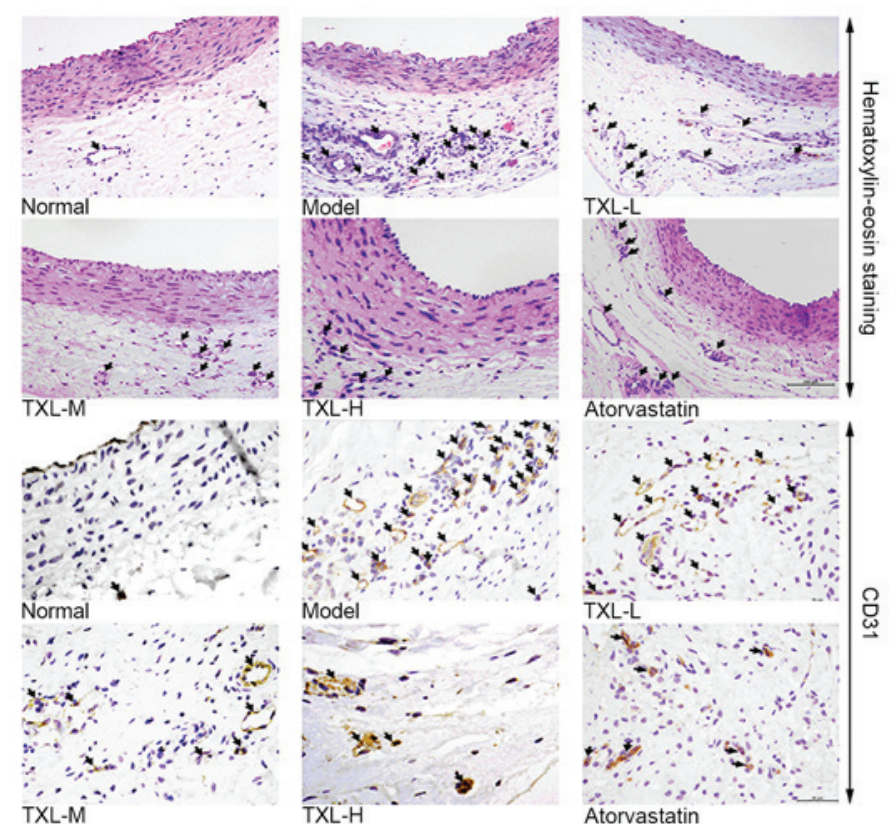

C
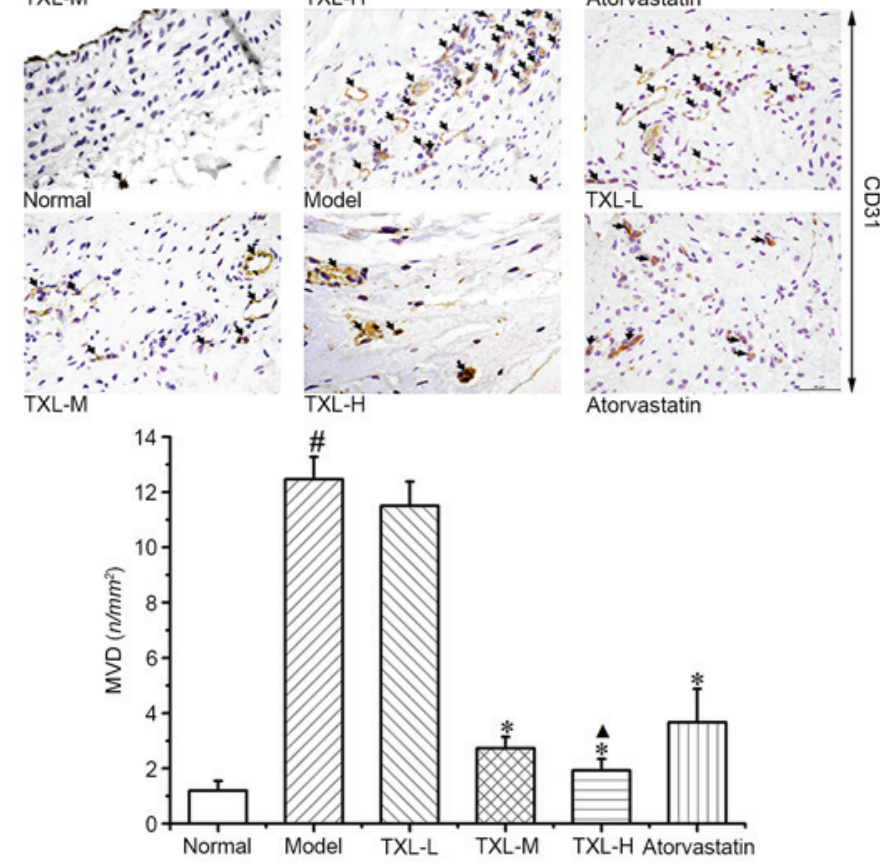

Figure 1. HE staining and CD31 immunohistochemical staining from 3 rabbits in each group. (A) HE-stained paraffin sections of the rabbit carotid arteries. Arterial wall changes with neointima and vasa vasorums are observed. Magnification, x200. (B) Representative micrographs demonstrating capillary density/CD31 in the different experimental groups. Magnification, x400. Arrows indicate the vasa vasorum in adventitia. (C) Quantitative analysis of capillary density in counts $/ \mathrm{mm}^{2}$. Data are presented as the mean \pm standard deviation. ${ }^{*} \mathrm{P}<0.01$ vs. normal. ${ }^{*} \mathrm{P}<0.01$ vs. model. ${ }^{\boldsymbol{}} \mathrm{P}<0.05$ vs. atorvastatin. HE, hematoxylin and eosin; TXL, tongxinluo; L, low; M, medium; H, high; MVD, microvessel density.

the mean \pm standard deviation. Prior to data analysis, normal distribution and homogeneity of variance were tested using D-test and F-test methods. For data with a normal distribution and homogenous variance, one-way analysis of variance was used for between-group comparison and the least significant difference was used for pairwise comparison. Differences in data with normal distribution and heterogeneous variance were tested using Dunnett's method. $\mathrm{P}<0.05$ was considered to indicate a statistically significant difference.

\section{Results}

Serum lipid levels. Blood serum TC, TG, LDL and HDL levels of the rabbits in the model group were significantly higher 
A
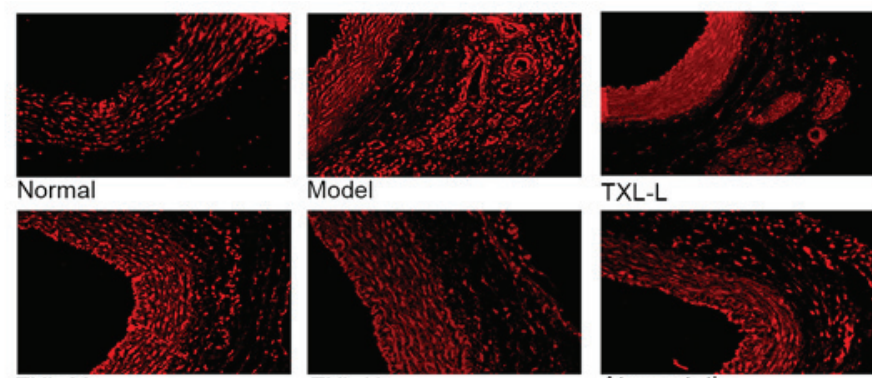

Model

TXL-L
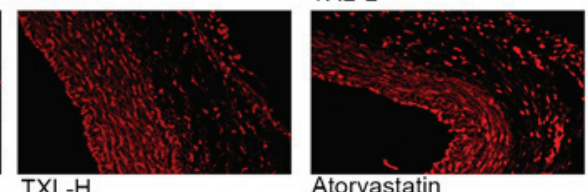

B
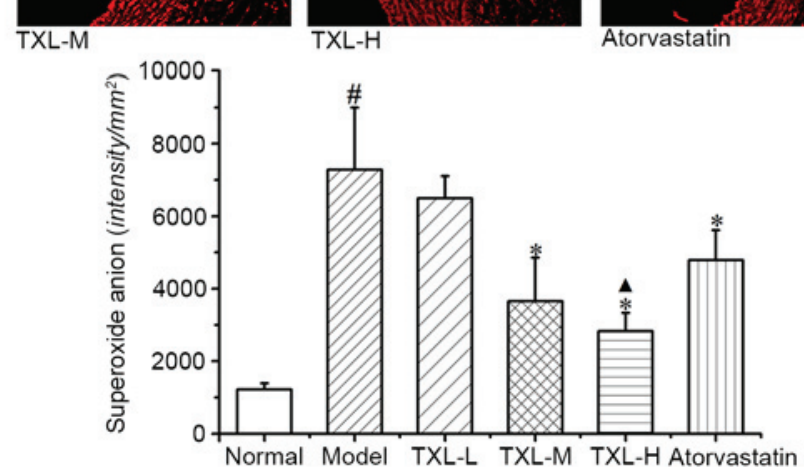

Figure 2. In situ detection of superoxide anion in carotid arteries. (A) Fluorescence micrographs of carotid arteries stained with the $\mathrm{O}_{2}^{-}$sensitive dye dihydroethidium (red fluorescence) were obtained at 28 days. Images of the carotid arteries were acquired at identical settings for each group. Each image is representative of results from 3 different animals. Magnification, x200. (B) Histogram of the quantitative analyses. Data are presented as the mean \pm standard deviation. ${ }^{\text {}} \mathrm{P}<0.01$ vs. normal group; ${ }^{*} \mathrm{P}<0.01$ vs. model group; ${ }^{\mathbf{A}} \mathrm{P}<0.05$ vs. atorvastatin group. TXL, tongxinluo; L, low; M, medium; H, high.

compared with those in the normal group $(\mathrm{P}<0.002$; Table I). Serum TC, TG and LDL levels in the TXL-H $(\mathrm{P}<0.004)$, TXL-M $(\mathrm{P}<0.047)$ and atorvastatin $(\mathrm{P}<0.007)$ groups were significantly lower compared with the model group (Table I). HDL levels in the TXL-H group and atorvastatin group were significantly higher compared with the model group $(\mathrm{P}<0.002$; Table I). No significant difference was identified between TC, TG, LDL and HDL levels in the TXL-H, TXL-M and atorvastatin groups (Table I).

Density of vasa vasorum in carotid arterial wall. As illustrated in Fig. 1, outward from the lumen, there were HE-positively stained cells in the intima, tunica media and adventitia with internal and external elastic membranes of carotid artery as boundaries. The tissues from the normal group exhibited smooth intima, continuous and complete monolayer of endothelial cells and a few vasa vasorum in the adventitia. In the model group, carotid neointima was formed and the quantity of vasa vasorum in the adventitia was increased. In the TXL-H, TXL-M and atorvastatin groups, intimal hyperplasia was reduced and the quantity of vasa vasorum in the adventitia was reduced to varying degrees.

The CD31 immunohistochemical staining demonstrated several CD31-positive microvessels in the carotid arteries of the normal group. The quantity of vasa vasorum was greater in the model group compared with the normal group. The TXL-L, TXL-M, TXL-H and atorvastatin groups demonstrated different degrees of decline in the quantity of vasa vasorum in the adventitia. Quantitative analysis demonstrated that the MVD-positive labeling index in the model group tissues was significantly greater compared with the normal group tissues ( $\mathrm{P}<0.006$; Fig. 1C). The MVD-positive labeling indexes in the TXL-H, TXL-M and atorvastatin group tissues were significantly lower compared with the model group tissues $(\mathrm{P}<0.003$;
Fig. 1C). The TXL-H group exhibited a significant decrease in the MVD-positive labeling index compared with the atorvastatin group $(\mathrm{P}<0.038$; Fig. 1C).

Superoxide production. As demonstrated in Fig. 2A, low $\mathrm{O}_{2}^{-}$levels were detected in the carotid intima, tunica media and adventitia of the normal group. In the model group, all layers of vessels exhibited increased $\mathrm{O}_{2}{ }^{-}$generation, primarily in the adventitia, the endothelium of vasa vasorum and the surrounding areas. Adventitia $\mathrm{O}_{2}^{-}$levels in the TXL-H, TXL-M, TXL-L and atorvastatin groups decreased to different extents.

According to quantitative analysis of fluorescence intensity, the model group exhibited significantly higher $\mathrm{O}_{2}{ }^{-}$fluorescence intensity in the adventitia compared with the normal group ( $\mathrm{P}<0.007$; Fig. 2B). The TXL-H, TXL-M and atorvastatin groups exhibited significantly lower levels of $\mathrm{O}_{2}{ }^{-}$fluorescence intensity in the adventitia compared with the model group tissues $\left(\mathrm{P}<0.002\right.$; Fig. 2B). The $\mathrm{O}_{2}^{-}$fluorescence intensity in the adventitia of the TXL-H group was significantly lower compared with the atorvastatin group ( $\mathrm{P}<0.041$; Fig. 2B).

Localization and expression of NADPH oxidase subunits p22phox and gp91phox mRNA in carotid artery. According to the results of the in situ hybridization localization experiments presented in Fig. 3A and B, the weak p22phox gene hybridization signal associated with its expression in carotid artery was confined to the adventitia in the normal group. The distribution of the gp91phox gene hybridization signal indicated that it was primarily expressed in the adventitia and to a lesser extent in the intima. However, the gp91phox gene hybridization signal detected in the adventitia was greater compared with p22phox. In all layers of the vessels in the model group, the p22phox and gp91phox gene hybridization signal associated with their expression was strongly positive and primarily distributed in the 
A

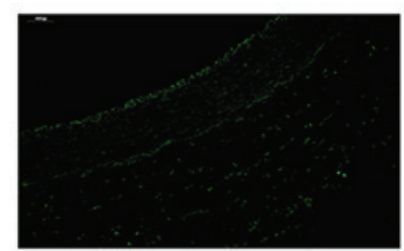

\section{Normal}

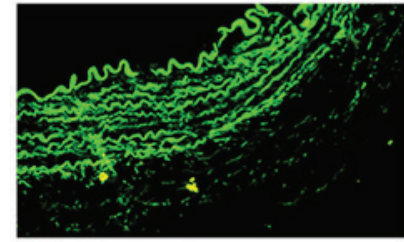

TXL-M

B

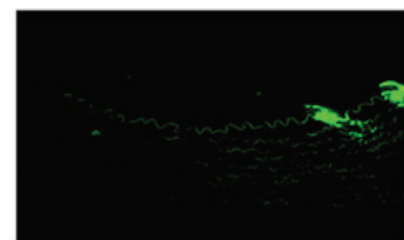

Normal

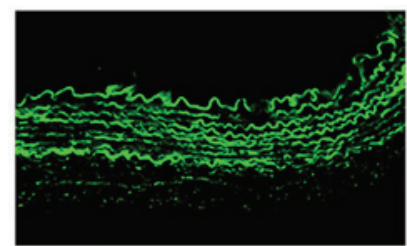

TXL-M

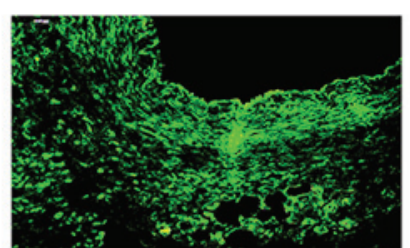

Model

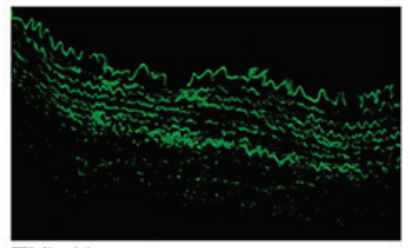

TXL-H

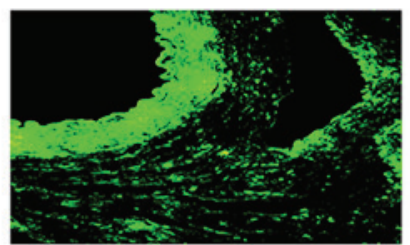

Model

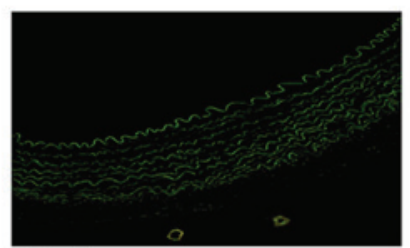

TXL-H

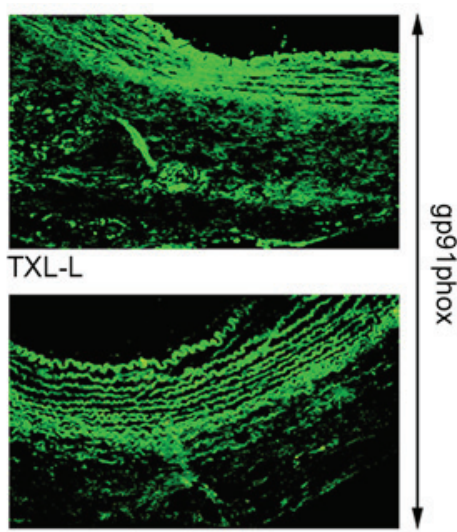

Atorvastatin

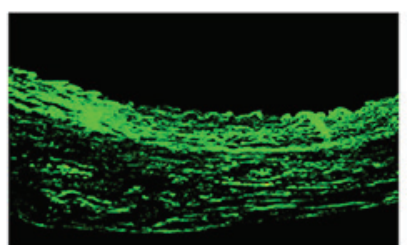

TXL-L

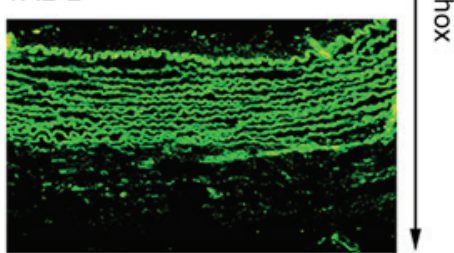

Atorvastatin

C

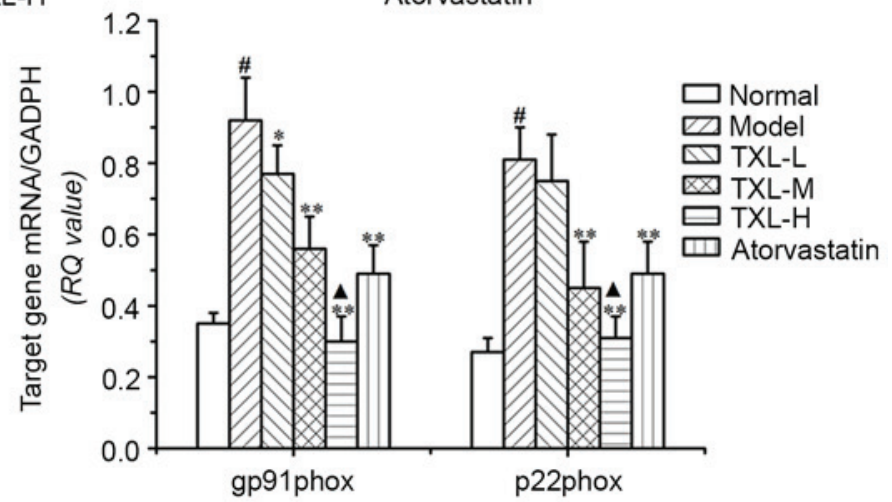

Figure 3. Localization and expression levels of the $\mathrm{NAD}(\mathrm{P}) \mathrm{H}$ oxidase subunits p22phox and gp91phox mRNA in carotid arteries. Each image is representative of results from 3 animals. (A) p22phox mRNA were localized primarily in the adventitia of normal arteries and in the adventitia, media and neointima of injured arteries. (B) gp91phox mRNA were localized to the adventitia or the intima in normal arteries and to the adventitia, media or neointima in injured arteries, detected by in situ hybridization. Magnification, x200. (C) Expression levels of p22phox and gp91phox mRNA of NAD(P)H oxidase subunits analyzed by reverse transcription-polymerase chain reaction in carotid arteries. Representative findings showing 77 and 442 bp products of p22phox and gp91phox mRNA. The histograms represent the ratios of the optical density of quantitative analyses. Densitometric analysis was performed, data are presented as the mean \pm standard deviation of 3 experiments. ${ }^{\text {}} \mathrm{P}<0.05$ vs. normal group; ${ }^{*} \mathrm{P}<0.05,{ }^{* *} \mathrm{P}<0.01$ vs. model group; ${ }^{\mathbf{4}} \mathrm{P}<0.05$ vs. atorvastatin group. $\mathrm{NAD}(\mathrm{P}) \mathrm{H}$, nicotinamide adenine dinucleotide phosphate; TXL, tongxinluo; L, low; M, medium; H, high.

intima and adventitia. No difference was identified between the expression of the p22phox and gp91phox genes in the TXL-L group and the model group. In the TXL-H, TXL-M and atorvastatin groups, the expression of p22phox and gp91phox were reduced in the adventitia.

For evaluating the effects of ROS induced NADPH oxidase were evaluated by analyzing, p22phox and gp91phox mRNA expression using RT-PCR analysis. It was determined that the p22phox and gp91phox mRNA expression levels in the model group were significantly higher compared with the normal group $(\mathrm{P}<0.001$; Fig. 3C). No significant difference was identified between the expression levels of p22phox and gp91phox mRNAs between the TXL-L group and the model group (Fig. 3C). The p22phox and gp91phox mRNA expression levels in the TXL-H, TXL-M and atorvastatin groups were significantly reduced compared with the model group $(\mathrm{P}<0.005$; Fig. $3 \mathrm{C})$. There was a significant reduction in the p22phox and gp91phox mRNA expression levels in the TXL-H group compared with the atorvastatin group $(\mathrm{P}<0.019$; Fig. 3C).

Relative protein expression levels of VEGF and VEGFR-2. The model group exhibited significantly greater protein 
A

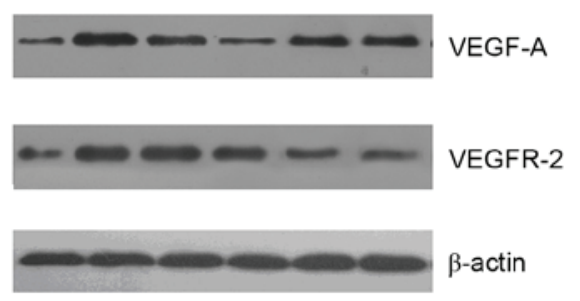

B

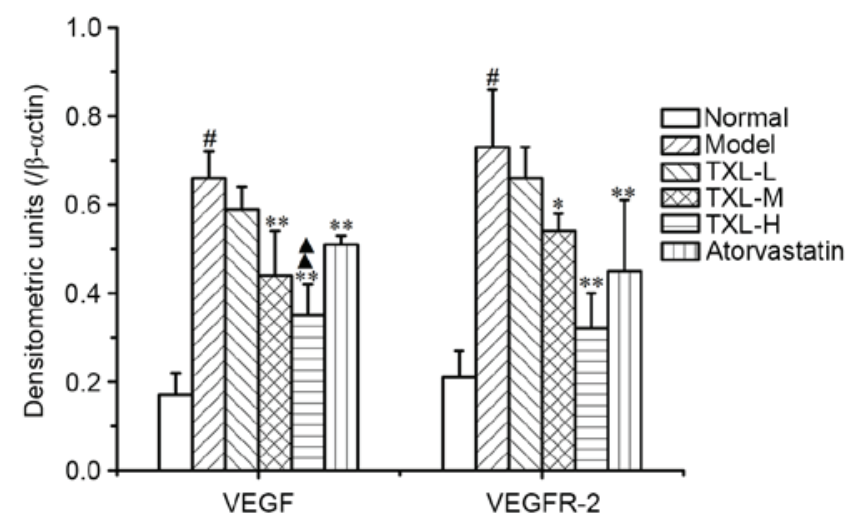

Figure 4. Western blot analysis of angiogenic factors in carotid arteries VEGF and VEGF-R2 expression levels were analyzed. (A) Representative western blots are presented for VEGF and VEGFR-2. Results are from 3 independent experiments. (B) Histograms represent the ratios of the optical density of quantitative analyses. Data are presented as the mean \pm standard deviation, and are expressed as the ratio to actin. ${ }^{\#} \mathrm{P}<0.05$ vs. normal group; ${ }^{*} \mathrm{P}<0.05,{ }^{* *} \mathrm{P}<0.01$ vs. model group; ${ }^{\Delta \boldsymbol{\Delta}} \mathrm{P}<0.01$ vs. atorvastatin group. VEGF vascular endothelial growth factor; VEGFR-2, vascular endothelial growth factor receptor-2; TXL, tongxinluo; L, low; M, medium; H, high.

expression levels of VEGF and VEGFR-2 in the carotid artery compared with the normal group $(\mathrm{P}<0.003$; Fig. 4). The TXL-H, TXL-M and atorvastatin groups demonstrated significantly lower VEGF protein expression levels compared with the model group $(\mathrm{P}<0.004$; Fig. 4). The relative VEGF protein expression level was significantly lower in the TXL-H group compared with the atorvastatin group $(\mathrm{P}<0.004$; Fig. 4). Additionally, the relative expression level of the VEGFR-2 protein in the TXL-M group was significantly lower compared with the model group $(\mathrm{P}<0.031$; Fig. 4). The VEGFR-2 expression levels in the TXL-H and atorvastatin groups were also significantly lower compared with the model group $(\mathrm{P}<0.008$; Fig. 4). The expression levels of the VEGFR-2 protein in the TXL-H group were reduced compared with the atorvastatin group, however, no significant difference was identified (Fig. 4).

\section{Discussion}

AS is a common arterial disease that seriously threatens human health. Additionally, it is the pathological basis underlying the development of cardiovascular and cerebrovascular diseases. Previous studies have demonstrated that pathological angiogenesis in the arterial wall is frequently observed during the atherosclerotic process (17-20). Angiogenesis is an important factor in the development of unstable atherosclerotic lesions (21). The results of the present study indicate that TXL inhibits adventitia angiogenesis. The moderate dose of TXL (TXL-M group) exerted an anti-angiogenic effect comparable with atorvastatin and the high-dose of TXL (TXL-H group) exhibited a greater anti-angiogenic effect when compared with atorvastatin, which was used as a positive control medicine in the present study, as it regulates angiogenesis. Previous studies have reported that atorvastatin treatment may decrease angiogenesis and suppress formation of plaques (22-24). It is possible that the anti-AS mechanism of TXL is associated with its inhibitory effect on angiogenesis. This may be due to its ability to reduce blood perfusion and flow velocity in the vasa vasorum, decreasing the exchanged endothelial area in vascular walls, thus preventing infiltration of pro-inflammatory and pro-arteriosclerotic blood components into the arterial wall; therefore, weakening the inflammatory reaction in the vascular wall (25-27). Anti-angiogenic treatment with TXL may be an effective AS treatment strategy.

Previous studies have identified ROS as regulators of cellular signaling and survival, with cell signaling and disease being investigated in in vivo and in vitro studies. Additionally, ROS involvement has been identified in pathological neovascularization (28-30). ROS may trigger various redox signaling pathways and lead to altered expression of angiogenesis-associated genes, migration and proliferation of endothelial cells, re-arrangement of the cytoskeleton (31) and the occurrence of tubular shape (32), which may further facilitate angiogenesis. The results of the present study indicated that compared with the model group, TXL treatment decreased ROS levels in adventitia in a dose-dependent manner and downregulated expression of the pro-angiogenic factors involved in the VEGF/VEGFR-2 signaling pathway. This indicated that the possible mechanism of inhibition of angiogenesis by TXL treatment in hyperlipidemic rabbits is associated with reduced adventitia ROS generation and the resulting activation of the downstream VEGF/VEGFR-2 signaling pathway. In angiogenesis, ROS may directly stimulate VEGF, which induces proliferation and migration of endothelial cells. Additionally, VEGF may activate NADPH oxidase, increase ROS generation (33), initiate VEGFR-2 autophosphorylation, provoke endothelial cell proliferation and migration and lumen formation; therefore, TXL may inhibit a positive feedback pathway involved in oxidative stress during angiogenesis.

In order to determine the effect of ROS on angiogenesis in the arterial wall, the mRNA levels of the NADPH oxidase subunits were detected. NADPH oxidase is a crucial enzyme involved in endovascular ROS generation in cardiovascular diseases (34). As a combined enzyme agent with multiple subunits, it is composed of heterodimers that include the membrane subunits p22phox and gp91phox, and several cytoplasmic protein components, including p47phoX, p67phox, p40phox and a small G protein, Rac1. gp91phox and p22phox are the core of NADPH oxidase (35). A previous study determined that NADPH oxidase is an important regulator of angiogenesis (36). To the best of our knowledge, the effects of p22phox and gp91phox on pathological angiogenesis in the arterial wall at the early stage of AS development have not been widely reported. The present study determined that during neovascularization, the levels of p22phox and gp91phox mRNA adjacent to the vasa vasorum and the carotid artery were increased. TXL reduced the p22phox and gp91phox expression levels in the carotid artery and adventitia. 
These results indicated that $\mathrm{p} 22$ phox and gp91phox may be important regulators of adventitia angiogenesis, and that TXL may reduce ROS generation by downregulating p22phox and gp91phox expression levels in adventitia. As demonstrated by a previous study, p22phox gene knock-out mice exhibited reduced tumor neovascularization (37). Additionally, during in vitro culture of endothelial cells, gene silencing of $\mathrm{p} 22 \mathrm{phox}$ partially limited ROS generation, reduced peroxisome proliferator-activated receptor expression and inhibited angiogenesis (37). In vitro experiments performed in a previous study determined that gp91phox was involved in endothelial cell proliferation, survival and that gp91phox gene silencing may influence endothelial cell morphology (38). Additionally, the hind limb ischemia model determined that gp91phox gene knock-out mice exhibited a delayed increase in capillary density. This mechanism may be associated with the inhibited combination of gp91phox with actin, IQ motif containing GTPase activating protein 1 and Racl binding protein, and the promotion of endothelial cell migration (39). The current study has determined that TXL may suppress neovascularization through the downregulation of p22phox and gp91phox gene expression in the adventitia.

In conclusion, the present study identified that TXL may inhibit adventitia angiogenesis in hyperlipidemic rabbits. This is potentially associated with the downregulation of adventitia ROS generation and the VEGF/VEGFR-2 signaling pathway. Therefore, TXL may be a potential therapeutic agent for treating hyperlipidemia and inhibiting angiogenesis. However, no direct causal association was observed in the present study and whether the observed effect is the cause or consequence of the anti-AS function of TXL has not been fully determined. The current study was performed using rabbits; however, human clinical trials should be conducted in the future in order to elucidate the effect of TXL on AS.

\section{References}

1. Li AC and Glass CK: The macrophage foam cell as a target for therapeutic intervention. Nat Med 8: 1235-1242, 2002.

2. Palinski W, Ord VA, Plump AS, Breslow JL, Steinberg D and Witztum JL: ApoE-deficient mice are a model of lipoprotein oxidation in atherogenesis. Demonstration of oxidation-specific epitopes in lesions and high titers of autoantibodies to malondialdehyde-lysine in serum. Arterioscler Thromb 14: 605-616, 1994.

3. Ross R: Atherosclerosis - an inflammatory disease. N Engl J Med 340: 115-26, 1999

4. Mitka M: Cholesterol drug lowers LDL-C levels but again fails to show clinical benefit. JAMA 303: 211-212, 2010.

5. Herrmann J, Lerman LO, Rodriguez-Porcel $M$, Holmes DR Jr, Richardson DM, Ritman EL and Lerman A: Coronary vasa vasorum neovascularization precedes epicardial endothelial dysfunction in experimental hypercholesterolemia. Cardiovasc Res 51: 762-766, 2001.

6. Staub D, Patel MB, Tibrewala A, Ludden D, Johnson M, Espinosa P, Coll B, Jaeger KA and Feinstein SB: Vasa vasorum and plaque neovascularization on contrast-enhanced carotid ultrasound imaging correlates with cardiovascular disease and past cardiovascular events. Stroke 41: 41-47, 2010.

7. Langheinrich AC, Michniewicz A, Sedding DG, Walker G, Beighley PE, Rau WS, Bohle RM and Ritman EL: Correlation of vasa vasorum neovascularization and plaque progression in aortas of apolipoprotein E(-/-)/low-density lipoprotein(-/-) double knockout mice. Arterioscler Thromb Vasc Biol 26: 347-352, 2006.

8. Tanaka K, Nagata D, Hirata Y, Tabata Y, Nagai R and Sata M: Augmented angiogenesis in adventitia promotes growth of atherosclerotic plaque in apolipoprotein E-deficient mice. Atherosclerosis 215: 366-373, 2011.
9. Gössl M, Herrmann J, Tang H, Versari D, Galili O, Mannheim D, Rajkumar SV, Lerman LO and Lerman A: Prevention of vasa vasorum neovascularization attenuates early neointima formation in experimental hypercholesterolemia. Basic Res Cardiol 104: 695-706, 2009.

10. Wilson SH, Herrmann J, Lerman LO, Holmes DR Jr, Napoli C, Ritman EL and Lerman A: Simvastatin preserves the structure of coronary adventitial vasa vasorum in experimental hypercholesterolemia independent of lipid lowering. Circulation 105: 415-418, 2002.

11. Moulton KS, Vakili K, Zurakowski D, Soliman M, Butterfield C, Sylvin E, Lo KM, Gillies S, Javaherian K and Folkman J: Inhibition of plaque neovascularization reduces macrophage accumulation and progression of advanced atherosclerosis. Proc Natl Acad Sci USA 100: 4736-4741, 2003.

12. Moulton KS, Heller E, Konerding MA, Flynn E, Palinski W and Folkman J: Angiogenesis inhibitors endostatin or TNP-470 reduce intimal neovascularization and plaque growth in apolipoprotein E-deficient mice. Circulation 99: 1726-1732, 1999.

13. Chen WQ, Zhong L, Zhang L, Ji XP, Zhao YX, Zhang C, Jiang H, Wu YL and Zhang Y: Chinese medicine tongxinluo significantly lowers serum lipid levels and stabilizes vulnerable plaques in a rabbit model. J Ethnopharmacol 124: 103-110, 2009.

14. Zhang L, Liu Y, Lu XT, Wu YL, Zhang C, Ji XP, Wang R, Liu CX, Feng JB, Jiang H, et al: Traditional Chinese medication Tongxinluo dose-dependently enhances stability of vulnerable plaques: A comparison with a high-dose simvastatin therapy. Am J Physiol Heart Circ Physiol 297: H2004-H2014, 2009.

15. Weidner N, Carroll P, Flax J, Blumenfeld W and Folkman J: Tumor angiogenesis correlates with metastasis in invasive prostate carcinoma. Am J Pathol 143: 401-409, 1993.

16. Purushothaman KR, Sanz J, Zias E, Fuster V and Moreno PR: Atherosclerosis neovascularization and imaging. Curr Mol Med 6: 549-56, 2006.

17. Roy H, Bhardwaj S, Babu M, Kokina I, Uotila S, Ahtialansaari T, Laitinen T, Hakumaki J, Laakso M, Herzig KH and Ylä-Herttuala S: VEGF-A, VEGF-D, VEGF receptor-1, VEGF receptor-2, NF-kappaB, and RAGE in atherosclerotic lesions of diabetic Watanabe heritable hyperlipidemic rabbits. FASEB J 20: 2159-61, 2006.

18. Moulton KS: Angiogenesis in atherosclerosis: gathering evidence beyond speculation. Curr Opin Lipidol 17: 548-55,2006.

19. Nakamura R, Sene A, Santeford A, Gdoura A, Kubota S, Zapata N, Apte RS: IL10-driven STAT3 signalling in senescent macrophages promotes pathological eye angiogenesis. Nat Commun 6: $7847,2015$.

20. Szöcs K, Lassègue B, Sorescu D, Hilenski LL, Valppu L, Couse TL, Wilcox JN, Quinn MT, Lambeth JD and Griendling KK: Upregulation of Nox-based NAD(P)H oxidases in restenosis after carotid injury. Arterioscler Thromb Vasc Biol 22: 21-27, 2002.

21. Moreno PR, Purushothaman KR, Fuster V, Echeverri D, Truszczynska H, Sharma SK, Badimon JJ and O'Connor WN: Plaque neovascularization is increased in ruptured atherosclerotic lesions of human aorta: Implications for plaque vulnerability. Circulation 110: 2032-2038, 2004.

22. Weis M, Heeschen C, Glassford AJ and Cooke JP: Statins have biphasic effects on angiogenesis. Circulation 105: 739-745, 2002.

23. Tian J, Hu S, Jia H, Hou J, Zhang S, Yu B and Jang IK: Correlation of vasa vasorum and plaque progression and response to atorvastatin therapy an a rabbit model of atherosclerosis: In vivo intravascular ultrasound and contrast-enhanced ultrasound imaging study. J Am Coll Card 59: E1072, 2012.

24. Bot I, Jukema JW, Lankhuizen IM, van Berkel TJ and Biessen EA: Atorvastatin inhibits plaque development and adventitial neovascularization in ApoE deficient mice independent of plasma cholesterol levels. Atherosclerosis 214: 295-300, 2011.

25. Fleiner M, Kummer M, Mirlacher M, Sauter G, Cathomas G, Krapf R and Biedermann BC: Arterial neovascularization and inflammation in vulnerable patients: Early and late signs of symptomatic atherosclerosis. Circulation 110: 2843-2850, 2004.

26. Moreno PR, Purushothaman KR, Sirol M, Levy AP and Fuster V: Neovascularization in human atherosclerosis. Circulation 113: 2245-2252, 2006.

27. Rademakers T, Douma K, Hackeng TM, Post MJ, Sluimer JC, Daemen MJ, Biessen EA, Heeneman S and van Zandvoort MA: Plaque-associated vasa vasorum in aged apolipoprotein E-deficient mice exhibit proatherogenic functional features in vivo. Arterioscler Thromb Vasc Biol 33: 249-256, 2013. 
28. Kim YW and Byzova TV: Oxidative stress in angiogenesis and vascular disease. Blood 123: 625-631, 2014.

29. Giacco F and Brownlee M: Oxidative stress and diabetic complications. Circ Res 107: 1058-1070, 2010.

30. Chen J, Liu B, Yuan J, Yang J, Zhang J, An Y, Tie L, Pan Y and Li X: Atorvastatin reduces vascular endothelial growth factor (VEGF) expression in human non-small cell lung carcinomas (NSCLCs) via inhibition of reactive oxygen species (ROS) production. Mol Oncol 6: 62-72, 2012.

31. Vepa S, Scribner WM, Parinandi NL, English D, Garcia JG and Natarajan V: Hydrogen peroxide stimulates tyrosine phosphorylation of focal adhesion kinase in vascular endothelial cells. Am J Physiol 277: L150-L158, 1999.

32. Shono T, Ono M, Izumi H, Jimi SI, Matsushima K, Okamoto T, Kohno K and Kuwano M: Involvement of the transcription factor NF-kappaB in tubular morphogenesis of human microvascular endothelial cells by oxidative stress. Mol Cell Biol 16: 4231-4239, 1996.

33. Giacca $M$ and Zacchigna S: VEGF gene therapy: Therapeutic angiogenesis in the clinic and beyond. Gene Ther 19: 622-629, 2012.

34. Griendling KK, Sorescu D and Ushio-Fukai M: NAD (P)H oxidase: Role in cardiovascular biology and disease. Circ Res 86: 494-501, 2000
35. Vignais PV: The superoxide-generating NADPH oxidase: Structural aspects and activation mechanism. Cell Mol Life Sci 59: 1428-1459, 2002

36. Konior A, Schramm A, Czesnikiewicz-Guzik M and Guzik TJ: NADPH oxidases in vascular pathology. Antioxid Redox Signal 20: 2794-2814, 2014

37. Garrido-Urbani S, Jemelin S, Deffert C, Carnesecchi S, Basset O, Szyndralewiez C, Heitz F, Page P, Montet X, Michalik L, et al: Targeting vascular NADPH oxidase 1 blocks tumor angiogenesis through a PPAR $\alpha$ mediated mechanism. PLoS One 6: e14665, 2011.

38. Ikeda S, Yamaoka-Tojo M, Hilenski L, Patrushev NA, Anwar GM, Quinn MT and Ushio-Fukai M: IQGAP1 regulates reactive oxygen species-dependent endothelial cell migration through interacting with Nox2. Arterioscler Thromb Vasc Biol 25: 2295-2300, 2005.

39. Coso S, Harrison I, Harrison CB, Vinh A, Sobey CG, Drummond GR, Williams ED and Selemidis S: NADPH oxidases as regulators of tumor angiogenesis: Current and emerging concepts. Antioxid Redox Signal 16: 1229-1247, 2012 . 